PROCEEDINGS OF THE

AMERICAN MATHEMATICAL SOCIETY

Volume 141, Number 1, January 2013, Pages 139-149

S 0002-9939(2012)11507-2

Article electronically published on May 16, 2012

\title{
THE LAST INCOHERENT ARTIN GROUP
}

\author{
DANIEL T. WISE
}

(Communicated by Alexander N. Dranishnikov)

Abstract. We prove that the Artin group $A(235)$ is not coherent.

\section{INTRODUCTION}

A group is coherent if all its finitely generated subgroups are finitely presented. Droms characterized coherent right-angled Artin groups [Dro87. Subsequently, Gordon gave a classification of coherent Artin groups that depends upon the incoherence of the Artin group $A(235)$ associated with the Coxeter group $H_{3}$. Whether or not $A(235)$ is coherent was left as an open problem [Gor04. In this paper, we prove that $A(235)$ is not coherent, confirming Gordon's suspicion and maintaining the simplicity of his classification.

In \$2. we review the incoherence of most three generator Artin groups, using our main tool: the Bestvina-Brady Morse theory. There appears to be a rather uniform source for the incoherence: There is a subcomplex $Y$ of the covering space of the standard 2-complex $X$ of the usual presentation of the Artin group $A$, and the Bestvina-Brady theorem applies to show that $\pi_{1} Y$ is not coherent. This method fails in two cases: The family of Artin groups $A(22 n)$, which are coherent, and the Artin group $A(235)$, where the author was unable to prove that $Y$ is $\pi_{1}$-injective.

In $\$ 3$ we examine the groups $A(233), A(234)$, and $A(235)$ more closely and consider the finite index subgroup $\widehat{A}$ which is the kernel of the homomorphism $A \rightarrow C$, where $C$ is the underlying Coxeter group. Using geometric arguments, we show that $\widehat{A}$ splits as a (bigonal) graph of groups where all vertex groups and edge groups are isomorphic to a group $B$ (depending on $A$ ), and we determine a presentation for $B$.

In $\$ 4$ we observe that this leads to hierarchies for $A(233)$ and $A(234)$, as the group $B$ has a very simple structure as a graph of free groups. In particular, it is easy to see from this that $\pi_{1} Y$ is a subgroup in this case. Our many efforts to prove something similar for $A(235)$ were stymied, and we have emerged with a healthy respect for $A(235)$.

In $\$ 5$, we use the center of $A$ to see that the splitting of $\widehat{A}$ is actually a product $B \times \mathbb{Z}$ - undoubtedly an old and basic fact, but one that we found rather surprising. We use this to see that $\operatorname{cd}(B)=2$, which we could not otherwise prove for $A(235)$. We then apply the Bestvina-Brady theorem to $B$ to obtain a finitely generated normal subgroup of $B$ that is not finitely presented. This proof works for $A(233)$,

Received by the editors September 8, 2010 and, in revised form, June 13, 2011.

2010 Mathematics Subject Classification. Primary 20F36, 20 E06.

Key words and phrases. Artin group, coherence.

This research was supported by NSERC. 

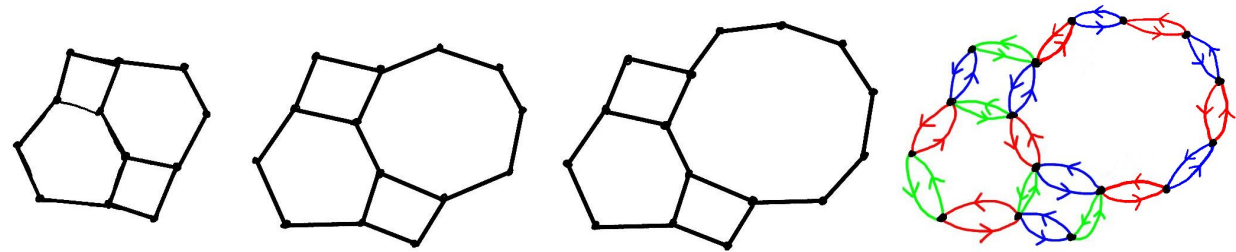

Figure 1. The 1-skeleton of the subcomplex $Y$ is sketched for $A(233), A(234)$, and $A(235)$ on the left, and is fully drawn for $A(235)$ on the right.

$A(234)$, and $A(235)$. We give an alternate proof that relies on a result of Bieri characterizing finitely presented normal subgroups, and this only works for $A(235)$.

We close the paper with a collection of problems in $\$ 6$.

\section{Applying Bestvina-Brady Morse theory}

In this section we describe a simple application of Bestvina-Brady Morse theory to detect finitely generated normal subgroups of subgroups of Artin groups. We refer the reader to [BB97] for the notion of Morse functions and ascending and descending links as applicable here. A short summary of the Bestvina-Brady theorem is:

Proposition 2.1. Given an equivariant Morse function $\tilde{Y} \rightarrow \mathbb{R}$ associated to a homomorphism $G \rightarrow \mathbb{Z}$, if the ascending and descending links are connected, then the kernel is finitely generated.

Moreover, if $\tilde{Y}$ is 2-dimensional and aspherical and some ascending or descending link is not contractible, then the kernel is not finitely presented.

We apply the Morse theory to a subcomplex $Y \subset \widehat{X}$, where $\widehat{X} \rightarrow X$ is the covering space corresponding to the Coxeter group quotient, and $X$ is the standard 2-complex of the three generator Artin group $A(p q r)$. Recall that $A(345)$ is presented by $\langle a, b, c \mid a b a=b a b, b c b c=c b c b, a c a c a=c a c a c\rangle$ and presentations for $A(p q r)$ are similar.

When $p, q, r \geq 3$, then the smallest example $Y$ to consider is associated to three polygons glued around a vertex and their lengths are $2 p, 2 q, 2 r$. When $p=2$ and $q, r \geq 3$, then we use the union of two polygons of lengths $2 q, 2 r$ glued along an edge, with additional squares attached along its two endpoints. We refer the reader to Figure 1, where we have illustrated the cases $A(233), A(234), A(235)$, and have illustrated the actual 1-skeleton of $Y$ for the $A(235)$ case.

Our "Morse function" map arises from the following commutative diagram, where $O$ is a circle having one 0 -cell and one oriented 1-cell, and the map $Y \rightarrow O$ is induced by the orientation-preserving combinatorial map $Y^{1} \rightarrow O^{1}$ :

$$
\begin{array}{ccc}
\tilde{Y} & \rightarrow & \widetilde{O} \\
\downarrow & & \downarrow \\
Y & \rightarrow & O
\end{array}
$$

We note that when $p, q=2$, the construction fails to yield incoherence, as the complex $Y$ does not $\pi_{1}$-inject in $\widehat{X}$. In fact $A(22 r)=\pi_{1} X$ is coherent in this case, since it is commensurable with $F_{2} \times \mathbb{Z}^{2}$. 

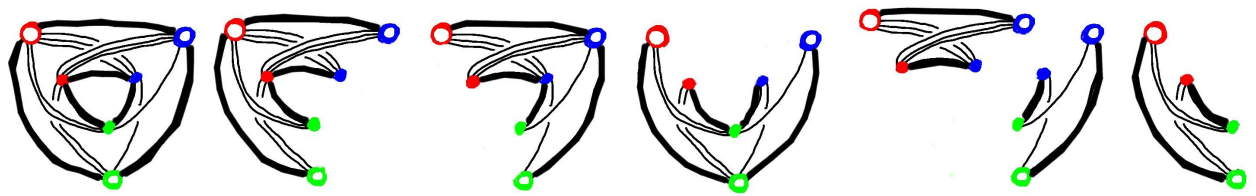

FiguRE 2. The various isomorphism types of links of 0-cells of $Y$ are illustrated for $A(234)$. Ascending and descending links are in bold.

We now observe that $Y$ is aspherical. One explanation is that it has a topological hierarchy whose edge spaces are $\pi_{1}$-injective graphs. This type of explanation is elaborated upon in 4

The kernel of $\pi_{1} Y \rightarrow \mathbb{Z}$ is finitely generated but not finitely presented. This follows from Proposition 2.1, since $Y$ is aspherical but the ascending and descending links are connected but not simply connected. See Figure 2 for the various ascending and descending links of $Y$ illustrated in the $A(234)$ case. It also follows from an idea perhaps first applied by Stallings: For a short exact sequence $1 \rightarrow N \rightarrow G \rightarrow Q \rightarrow 1$ we have $\chi(N) \chi(Q)=\chi(G)$, which is impossible in this case as $\chi(Q)=0$ but $\chi(G)>0$.

The map $Y \rightarrow \widehat{X}$ is readily shown to be $\pi_{1}$-injective for $A(p q r)$ with $\frac{1}{p}+\frac{1}{q}+\frac{1}{r} \leq 1$, but we do not describe the details, as we focus here on the spherical case. Except perhaps for $A(235)$, the map $Y \rightarrow \widehat{X}$ is $\pi_{1}$-injective since it factors as $Y \rightarrow V \rightarrow \widehat{X}$, where $V$ is a hemisphere vertex space (see $\$ 3$ ). The $\pi_{1}$-injectivity of the latter map is proven in $\$ 3$. For $A(233)$ and $A(234)$, the former map is $\pi_{1}$-injective since $Y$ can be sliced off from $V$ using a hierarchy.

\section{Great Circle splitting}

Let $A$ be a spherical Artin group, so $A$ is one of: $A(22 n), A(233), A(234), A(235)$. Let $X$ denote the standard 2-complex of the presentation of $A$.

Consider the quotient $A \rightarrow C$ to the underlying Coxeter group $C$. Let $\widehat{X} \rightarrow X$ denote the covering space associated to this quotient, so $\pi_{1} \widehat{X}$ is the kernel of $A \rightarrow C$. The group $C$ acts as a reflection group on the 2-sphere $S$ and there is an equivariant $\operatorname{map} \widehat{X} \rightarrow S$.

Theorem 3.1. For each reflection of $C$, let $W$ denote the associated great circle of $S$. Let $W_{1}, W_{2}$ denote the two components of the preimage of $W$ in $\widehat{X}$. Let $U, V$ denote the subcomplexes contained in the preimages of the components of $S-W$. Then $U \rightarrow \widehat{X}$ and $V \rightarrow \widehat{X}$ are $\pi_{1}$-injective. $\pi_{1} \widehat{X}$ splits as a graph of groups whose underlying graph is a bigon and whose edge groups map to vertex groups by isomorphisms. The vertex groups are isomorphic to $\pi_{1} U$ and $\pi_{1} V$, and the edge groups are the images of $\pi_{1} W_{1}$ and $\pi_{1} W_{2}$ in $\pi_{1} \widehat{X}$.

To see that the preimage of $W$ in $\widehat{X}$ consists of two components, note that $W_{1}$ passes through 1-cells that are consistently oriented from $U$ to $V$, and $W_{2}$ passes through 1-cells oriented from $V$ to $U$. Such pairs of graphs that are preimages of (arcs of) a great circle will be important again later; see Figure 12

Remark 3.2. I suspect Theorem 3.1 can be restated to hold more generally, possibly for all Artin groups. In particular, a modified version holds for planar-type Artin groups. 


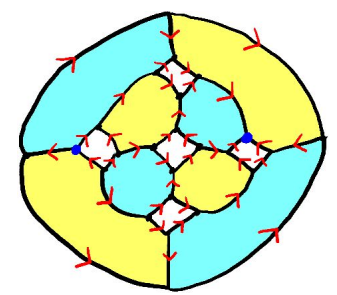

Figure 3. All edges are directed from the initial vertex to the terminal vertex.

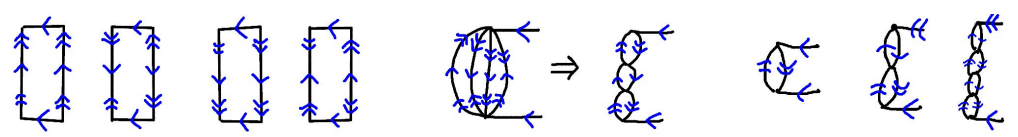

FIGURE 4. Folding shows that the arcs on one side of an Artin relator, freely generate a subgroup associated to an immersed chain of bigons.

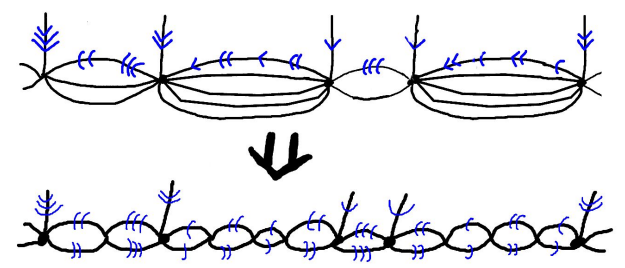

Figure 5. Folding one side of $\partial N_{(-1,1)}\left(W_{i}\right)$.

Definition 3.3 (Spherical section). There are various spherical sections $S \rightarrow \widehat{X}$ of the map $\widehat{X} \rightarrow S$. Each section $S=S_{v}$ corresponds to a choice $v$ of initial vertex by directing all 1-cells outwards from this initial vertex $v$ to an antipodal terminal vertex. Note that the 1-cells of $S$ are already labeled by the Coxeter group generators but are not directed. The 2-cells of a section $S$ are magically labeled and directed so that they agree with the Artin relators. We refer the reader to Figure 3 for a simple example.

Proof of Theorem 3.1. We will find that a sufficient set of relators for the subgroup of $\pi_{1} \widehat{X}$ generated by $\pi_{1} W_{i}$ is given by the spherical sections $S \rightarrow \widehat{X}$. Each such section overlaps $W_{i}$ along a great circle, and its two hemispheres naturally provide relators in the two vertex spaces $U, V$ of $\widehat{X}$.

Consider the open combinatorial neighborhood $N_{(-1,1)}\left(W_{i}\right)$ of $W_{i}$ consisting of all open cells intersecting $W_{i}$. We have $N_{(-1,1)}\left(W_{i}\right) \cong W_{i} \times(-1,1)$, and so identifying $W_{i}$ with $W_{i} \times\{0\}$ we see that $\pi_{1} W_{i}$ is isomorphic to $\pi_{1} N\left(W_{i}\right)$. As in Figures 4 and 5 , there is a $\pi_{1}$-isomorphism between $W_{i}$ and the graph $\partial V$ obtained by folding $W_{i} \times\{-1\}$ regarded as a component of the boundary of $W_{i} \times(-1,1)$. (Here we regard $V$ as being on the negative side of $W$.) We will use this below where we consider instead the half-open neighborhood $N_{[-1,0]}\left(W_{i}\right) \subset \widehat{X}$ which contains $W_{i}$ on one side and its folded image $\partial V$ on the other.

Let $Z=W_{i} \cup \bigcup_{v \in V^{0}}\left\{H_{v}\right\}$ denote the space obtained from $W_{i}$ by attaching a copy $H_{v}$ of the $V$-hemisphere of each section $S_{v}$. There is a natural map $H_{v} \rightarrow D_{v}$ that sends the closed disc $H_{v}$ to the disc diagram $D_{v}=\left(S_{v} \cap V\right)$ which consists of 
the subcomplex of $S_{v}$ consisting of all cells entirely contained within $V$. There is thus a map $Z \rightarrow V$, and we let $M$ denote the mapping cylinder of $Z \rightarrow V$.

We will prove that $Z \rightarrow V$ is a $\pi_{1}$-isomorphism by showing that (after removing some open 3-balls) the space $M$ deformation retracts to $Z \cup \bigcup_{W_{i}} N_{[-1,0]}\left(W_{i}\right)$ which has the same homotopy type as $Z$. An analogous explanation confirms the $\pi_{1^{-}}$ isomorphism $Z \rightarrow U$ as well as two additional such $\pi_{1}$-isomorphisms to $U, V$. It follows that $\pi_{1} \widehat{X}$ splits as a bigonal graph of groups and hence $U$ and $V$ are $\pi_{1}$ injective subcomplexes.

Collapsing faces of 3-balls: We begin with a sequence of (removed and) collapsed 3-balls of $M$ along free faces consisting of 2-cells in $V$. The sequence of collapses is indexed in order by numbers, but the exact order of the collapses is interchangeable when the indices are the same. A straight arrow indicates which 2-cell is the free face, and the basepoint of the arrow is the initial vertex of the section associated to the hemisphere collapse. See the first two diagrams in Figure 6. For each such collapse, we first remove all other open 3-balls attached along the (designated) free face 2-cell and then collapse by removing the free face 2-cell together with the final 3-ball. The reader can verify the critical feature of the ordered sequences: The 2-cells used as free faces of earlier 3-collapses do not occur on the attaching maps of subsequently collapsed 3-balls, and likewise the 1-cells used as free faces of earlier 2-collapses do not occur on the attaching maps of subsequently collapsed 2-balls.

Collapsing faces of 2-balls: A further sequence of 2-collapses of 2-cells along 1-cells is given in the subsequent diagrams using a different notation. The initial point of a "turned arrow" indicates the 2-cell that a 1-cell is collapsed along. The arrow proceeds from the corner along a 1-cell to indicate the free face where the collapse occurs. We refer the reader to the third and fourth diagrams in Figure 6 .

Collapsing faces of 1-balls: There is a final sequence of 1-collapses until we arrive at $Z \cup \bigcup_{W_{i}} N_{[-1,0]}\left(W_{i}\right)$. These are indicated by the trees in Figure 11.

We refer the reader to Figure 7 for the data describing the procedure for $A(233)$, and to Figures 9 and 10 for $A(234)$ and $A(235)$. While these figures are drawn on the modified Cayley graph of the associated Coxeter group, they must be interpreted for $\widehat{X}$ and actually for $M$. We recommend the reader first practice interpreting the data with $A(233)$. As there are two types of walls, there are essentially two combinatorial possibilities for both $A(22 n)$ and for $A(234)$. We leave $A(22 n)$ to the reader, and we indicate both possibilities for $A(234)$.

Remark 3.4. It seems that hyperbolic or Euclidean planes (instead of spheres) should be interpreted to mean that there are no additional relations. It would be interesting to see a formal proof generalizing the spherical situation.

\section{Hierarchies For the Vertex Groups}

We now explain that for $A(233)$ and $A(234)$ the vertex groups split as a graph of free groups. See Figure 12. Namely, for each arc of a great circle, there is a splitting as a graph of groups with two edge groups and two vertex groups. This is again induced by the splitting of the space as a graph of spaces, whose edge spaces are the carriers of the two components of the preimage of a great circle arc and whose two vertex spaces are the components of their complement. 

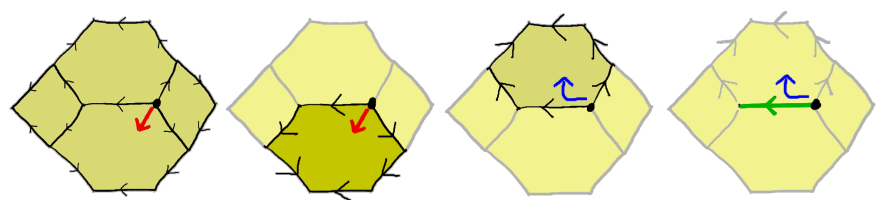

Figure 6. The straight arrow on the left indicates a 3-collapse, whose 3 -cell is attached along the hemisphere indicated by the arrowed half-section in the first diagram and whose free face is the highlighted 2-cell in the second diagram. The turned arrow on the right indicates a 2-collapse, whose 2-cell is highlighted in the third diagram, and whose free face is the highlighted 1-cell in the fourth diagram. In each case, the relevant cells are associated to attaching maps directed outwards from the basepoint of the arrow.

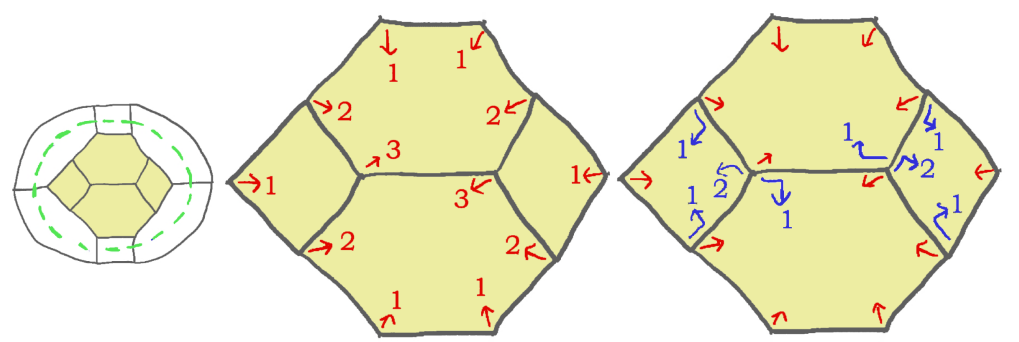

Figure 7. The wall $W$ is suggested on the left. There are three layers of 3-collapses indicated in the middle. The final sequence of 2 -collapses is indicated at the right. We have not indicated 1collapses.
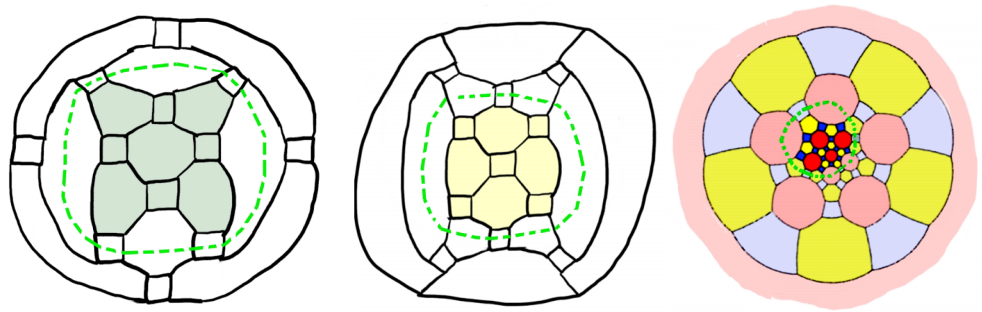

Figure 8. The subcomplex $V \subset \hat{X}$ is indicated for both variants of the 234 case and for the 235 case.

In this case, at each stage, the edge groups are free groups, and split geometrically as free products of the free groups one gets in this way by virtually splitting a single 2-generator Artin group.

I had originally expected $A(235)$ to split as a graph of graph-groups, but determining such a splitting (if it exists) will require ingenuity. See Figure 13 We nevertheless have:

Remark 4.1. The group $\pi_{1} V$ splits as a graph of groups whose underlying graph is a chain of four bigons. All edge groups and vertex groups are finitely generated, and all edge group attaching maps are isomorphisms.

This follows along the lines illustrated in Figure 13 . The four pairs of edge spaces associated to each bigon correspond to the four bold vertical curves. (Note that 

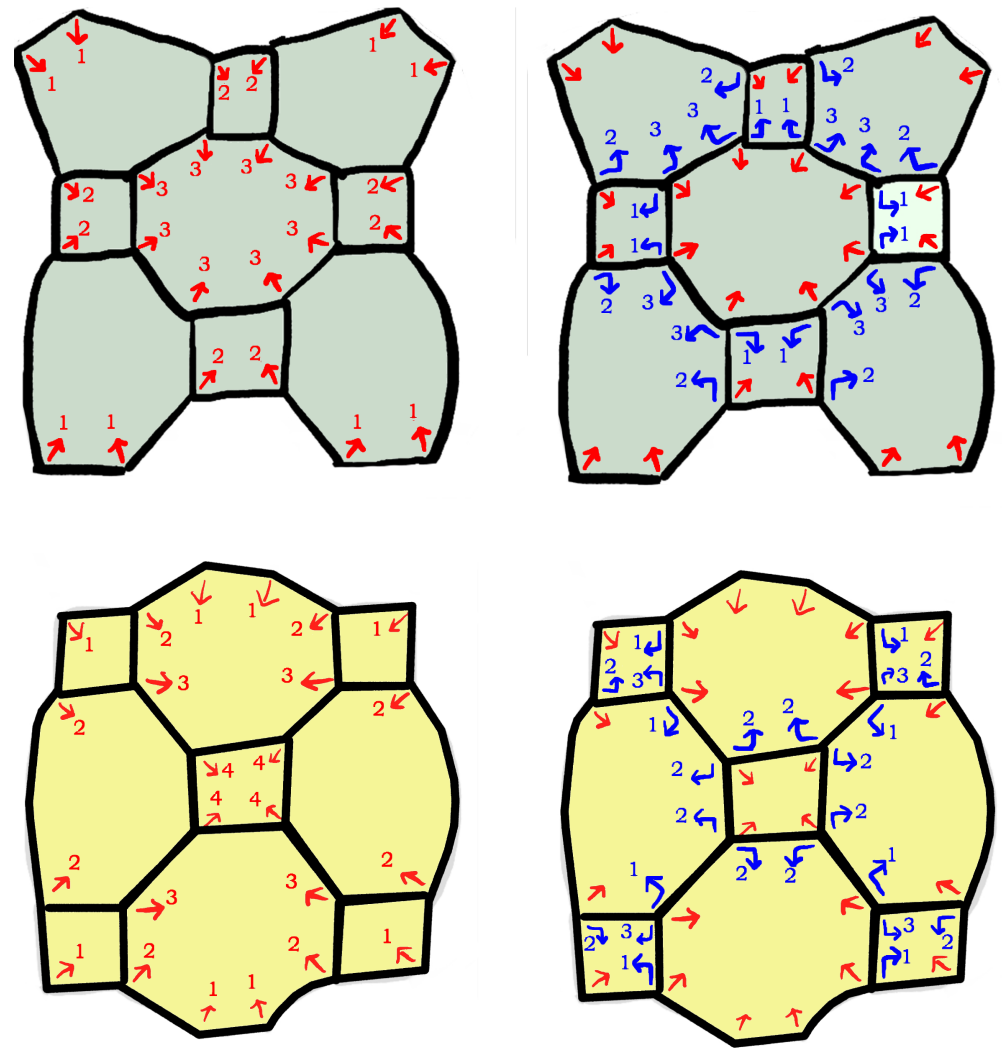

FiguRE 9. Both variants of the proof that $Z \rightarrow V$ is a $\pi_{1^{-}}$ isomorphism for $A(234)$. On the left is the first sequence of collapses, and on the right is the second sequence of collapses.

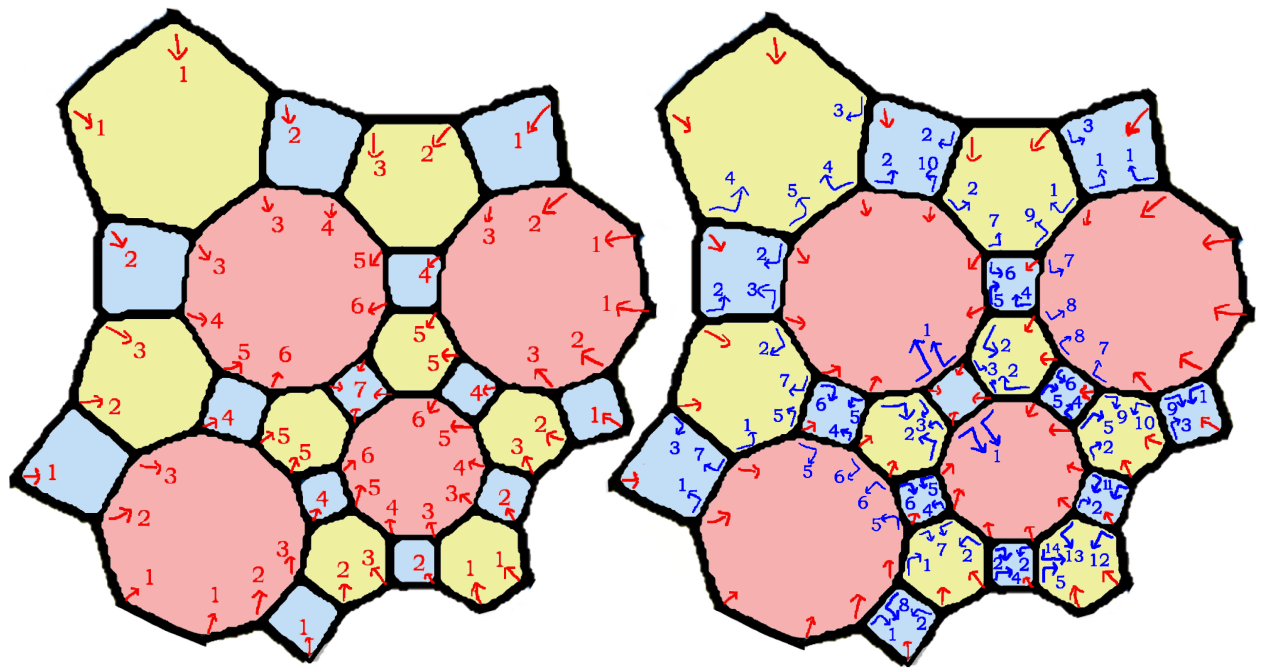

Figure 10. The 3-collapses and 2-collapses for the case of $A(235)$. 

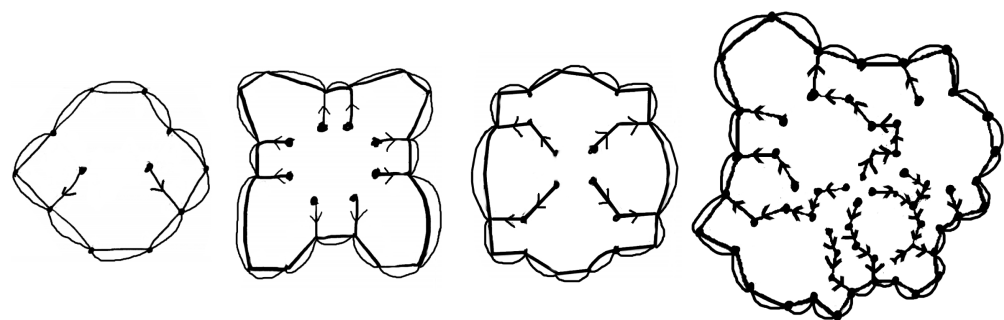

FiguRE 11. The remaining 1-collapses are determined by the trees. Our ad hoc choice of 2 - and 3 -collapses in the $A(235)$ case resulted in disorganized trees.
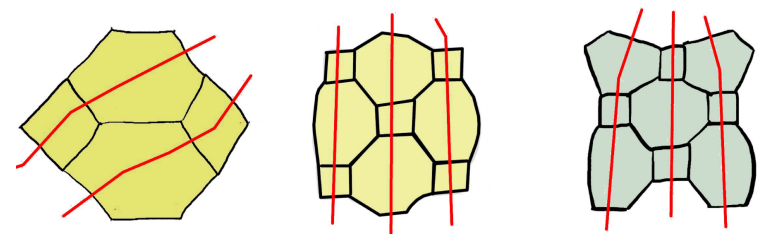

FIGURE 12. The splitting as a graph of free groups is induced by the remaining arcs of walls.

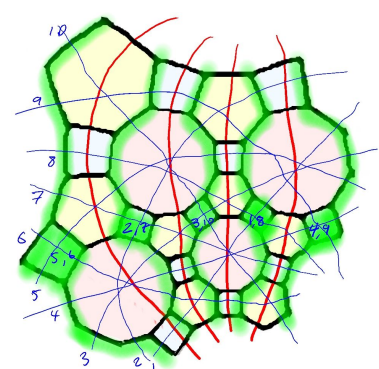

FiguRE 13. We were unable to find a simple combinatorial proof of asphericity of the subcomplex $V$ associated to the above diagram.

there is another such family of four curves associated to a second such splitting.) It is easy to check that the attaching maps of the edge spaces are $\pi_{1}$-surjective, and the result therefore follows.

\section{Cohomological Dimension, the Center, And the CONClusion}

The finite-type Artin groups with three generators are shown to act cocompactly on 3-dimensional CAT $(0)$ complexes in Bra00. In particular, this shows that they have cohomological dimension at most 3. For $\frac{1}{p}+\frac{1}{q}+\frac{1}{r}>1$, the group $A(p q r)$ contains a copy of $\mathbb{Z}^{3}$ and hence has cohomological dimension 3 .

Let $B=B(p q r)$ denote $\pi_{1} V$, where $V \subset \widehat{X}$ is the $\pi_{1}$-injective subcomplex contained in the hemisphere that we studied in $\S 3$. Let $\widehat{A}=\pi_{1} \widehat{X}$. As shown in Theorem [3.1. $\widehat{A}$ splits as a graph of groups where each vertex group is isomorphic to $B$, the underlying graph is a bigon, and the inclusions of edge groups are isomorphisms. Thus $\widehat{A} \cong B \rtimes \mathbb{Z}$. 
We will utilize the following striking property of finite-type Artin groups proven by Brieskorn-Saito and Deligne Del72].

Proposition 5.1. Let $\Delta$ denote a positive path in $S^{2}$ from the basepoint to the antipodal point. Then $\Delta^{2}$ represents an element in the center of $A$.

Observe that we can choose $\Delta^{2}$ as the generator of the $\mathbb{Z}$ factor in our decomposition of $\widehat{A}$ as $B \rtimes \mathbb{Z}$. Indeed the path $\Delta^{2}$ travels from a basepoint in the first copy of $V$ through the outward oriented edge space, and then returns through the inward oriented edge space and ends on the basepoint.

Applying Proposition 5.1 to see that $\Delta^{2}$ is central, we find that $\widehat{A} \cong B \times \mathbb{Z}$.

Consequently, $B$ has cohomological dimension $2 \operatorname{as~} \operatorname{cd}(A)=\operatorname{cd}(\widehat{A})=\operatorname{cd}(B)+1$. Here the first equality holds since $\widehat{A}$ is a finite index subgroup of the torsion-free group $A$, and the second equality holds since $\widehat{A} \cong B \times \mathbb{Z}$ Bro94.

The incoherence of the nonspherical three generator Artin groups was sketched in $\$ 2$, and we now reach our conclusion that the spherical Artin groups $A(p q r) \neq$ $A(22 n)$ are incoherent. In particular, this applies to $A(235)$ to obtain our main goal.

Theorem 5.2. Each of $A(233), A(234)$, and $A(235)$ has a (finite index subgroup with a normal) finitely generated subgroup that is not finitely presented.

We give two similar proofs yielding two finitely generated subgroups that are not finitely presented. The first proof applies uniformly, and the second only applies to $A(235)$. Apparently, the difficulty leading to the $A(235)$ gap in our earlier proof of incoherence (using $Y$ ) makes its presence felt here in additional incoherence.

The first proof uses the following fundamental result of Bieri Bie76].

Proposition 5.3. Let $G$ be a finitely generated group with $c d(G)=2$. Suppose that $N$ is a finitely presented normal subgroup of $G$. Then either $N$ is of finite index or $N$ is trivial or $N$ is free and $G / N$ is virtually free.

First proof of Theorem 5.2. We have shown that $\operatorname{cd}(B)=2$. Each torus $T$ corresponding to a square in the picture of $V \pi_{1}$-injects since it corresponds to an index 4 subgroup of a conjugate of a sub-Artin group.

The splitting of Remark 4.1 gives a decomposition $B \cong L \rtimes F$, where $F$ is free and $L$ is finitely generated. The images of $\pi_{1} T$ are conjugate into $L$, so we see that $L$ is not free. Applying Proposition 5.3 , we see that $L$ is not finitely presented.

Second proof of Theorem 5.2. It is easily computed that $\chi(V)>0$, which suggests that $\chi(B)>0$, but we have not shown that $V$ is aspherical. Nevertheless, we see that $\chi(B)>0$ as follows:

A routine computation (that is simplified by viewing $B$ as $L \rtimes F_{4}$ with $L 10$ generated) shows that $\beta_{1}(B) \leq 14$. For each $n$-gon in the picture of $V$, there is a retraction onto the corresponding subcomplex.

This gives retractions of $B$ onto various subgroups and hence provides embeddings of their homology groups, and the retractions show that their embedded $\mathrm{H}_{2}$ subgroups are independent. Each has $\beta_{2}=\frac{n}{2}-1$, and thus, since there are 13 4-gons, 8 6-gons, and 4 10-gons, we obtain $\beta_{2} \geq 13(1)+8(2)+4(4)=45$. In conclusion, we find that $\chi(B)=\beta_{2}-\beta_{1}+\beta_{0} \geq 45-14+1=32$. (Here we use that $\beta_{d}=0$ for $d \geq 3$.) 
Proposition 2.1 applies to the orientation induced map $V \rightarrow S^{1}$ to see that the kernel $K$ of $B \rightarrow \mathbb{Z}$ is finitely generated. Since $\chi(B)>0$ we can apply the Stallings contradiction described in $\$ 2$ to see that $K$ is not finitely presented. (Alternately, if $K$ were finitely presented, then Proposition 5.3 would imply that $K$ is free. However, I do not see a quick explanation that it is not free.)

\section{Problems}

Problem 6.1. Prove that the embedding $Y \subset V$ is $\pi_{1}$-injective for $A(235)$.

Problem 6.2. Give a direct proof that $\operatorname{cd}(B)=2$ for $A(235)$. Better yet, perhaps there is a combinatorial proof that $V$ is aspherical.

Remark 6.3. While the vertex groups of the obvious splittings of $B(235)$ are finitely generated, and indeed $B$ has an infinite finitely generated free quotient with a finitely generated kernel $K$, the subgroup $K$ is not finitely presented. Indeed it is not free as it contains a copy of $\mathbb{Z}^{2}$. It therefore cannot be finitely presented by Proposition 5.3.

Problem 6.4. Find a hierarchy for $B(235)$. Does it have a splitting as a graph of free groups? A graph of graph groups?

Problem 6.5. Find (virtual) hierarchies for arbitrary Artin groups. In particular, this appears to be approachable when the underlying Coxeter group is 2dimensional.

An interesting virtual hierarchy for $A(233)$ is given in [DLS91, and some other work giving hierarchies for $A(p q r)$ with $\frac{1}{p}+\frac{1}{q}+\frac{1}{r}=1$ was done in [Squ87. I expect there are many other valuable results in this direction by researchers obtaining such hierarchies with other goals in mind. Bestvina explicitly asked whether every finite-type Artin group is virtually poly-free Bes99.

Problem 6.6. Is there an injection $F_{2} \times F_{2} \hookrightarrow A(235)$ ?

Gordon explains why $F_{2} \times F_{2}$ cannot embed in $A(233)$ and $A(234)$ Gor04]. He attributes the original proof that it does not embed in $A(233)$ to Akimenkov.

\section{ACKNOWLEDGEMENT}

The author is grateful to the referee for comments that improved the exposition of this paper.

\section{REFERENCES}

[BB97] Mladen Bestvina and Noel Brady, Morse theory and finiteness properties of groups, Invent. Math. 129 (1997), no. 3, 445-470. MR1465330 (98i:20039)

[Bes99] Mladen Bestvina, Non-positively curved aspects of Artin groups of finite type, Geom. Topol. 3 (1999), 269-302 (electronic). MR1714913 (2000h:20079)

[Bie76] Robert Bieri, Normal subgroups in duality groups and in groups of cohomological dimension 2, J. Pure Appl. Algebra 7 (1976), no. 1, 35-51. MR0390078 (52:10904)

[Bra00] Thomas Brady, Artin groups of finite type with three generators, Michigan Math. J. 47 (2000), no. 2, 313-324. MR.1793627 (2001j:20056)

[Bro94] Kenneth S. Brown, Cohomology of groups, Springer-Verlag, New York, 1994, corrected reprint of the 1982 original. MR1324339 (96a:20072)

[Del72] Pierre Deligne, Les immeubles des groupes de tresses généralisés, Invent. Math. 17 (1972), 273-302. MR0422673 (54:10659) 
[DLS91] Carl Droms, Jacques Lewin, and Herman Servatius, Tree groups and the 4-string pure braid group, J. Pure Appl. Algebra 70 (1991), no. 3, 251-261. MR1104843 (92b:20040)

[Dro87] Carl Droms, Graph groups, coherence, and three-manifolds, J. Algebra 106 (1987), no. 2, 484-489. MR88e:57003

[Gor04] C. McA. Gordon, Artin groups, 3-manifolds and coherence, Bol. Soc. Mat. Mexicana (3) 10 (2004), Special Issue, 193-198. MR2199348 (2006i:57003)

[Squ87] Craig C. Squier, On certain 3-generator Artin groups, Trans. Amer. Math. Soc. 302 (1987), no. 1, 117-124. MR887500 (88g:20069)

Department of Mathematics and Statistics, McGill University, Montreal, Quebec, CANADA H3A 2K6

E-mail address: wise@math.mcgill.ca 